\title{
Collimation and decoupling of ECR source beams for brilliance optimization*
}

\author{
C. Xiao ${ }^{\dagger 1}$, L. Groening ${ }^{1}$, and O.K. Kester ${ }^{1,2}$ \\ ${ }^{1}$ GSI, Darmstadt, Germany; ${ }^{2}$ Goethe-Universität, Frankfurt am Main, Germany
}

\begin{abstract}
The four-dimensional transport of the transverse phase space of the extracted beam was calculated for the CAPRICE electron cyclotron resonance (ECR) ion source at GSI. The axial magnetic field adds an angular momentum to the extracted beam, resulting in a strongly $x-y$ coupled beam. The report presents multi-particle tracking simulations, and the results illustrate that the beam brilliance can be improved by combination of multi-stage collimation with skew quadrupole decoupling.
\end{abstract}

\section{Quadrupole Collimation Channel}

The quadrupole collimation channel is placed behind the analyzing magnet in order to improve the beam brightness. An uncorrelated beam with desired Twiss parameters is assumed at the entrance of the quadrupole collimation channel being the periodic matched solution of the channel. Only particles in a defined phase space volume are transmitted through the entire channel, all other particles are stopped at the apertures along the channel. The magnetic quadrupole collimation channel has three cells including three identical magnetic quadrupole doublets and four fourjaw slits. For efficient and flexible collimation, each cell is set to cause a phase space rotation of 45 degree and multiple cells with overall phase advance rotation larger than 90 degree (145 degree in this channel). Four successive four-jaw slits are used for multi-stage collimation with the phase space rotation in between [1].

\section{Skew quadrupole decoupling section}

After charge-to-mass selection, two normal quadrupole doublets are used to match the analyzed beam into the quadrupole collimation channel. The quadrupole collimation channel, which consists of three normal quadrupole doublets with the same gradient but alternating sign are used to carry out the multiple-stage phase space rotation. The decoupling section comprises two normal quadrupole doublets and one skew quadrupole triplet, and their gradients are optimized by a numerical routine to remove the inter-plane correlations, thus minimizing the product of the horizontal and vertical rms emittances [2].

* Work supported by the Helmholtz International Center for FAIR and the Bundesministerium für Bildung und Forschung.

†c.xiao@gsi.de

\section{Collimation and decoupling simulation}

In general, an ECR ion source beam possesses a large beam size and divergence. Therefore, higher order effects (aberrations) can not be avoided inside the solenoid. If the particle deviates from the center of the solenoid, it feels a non-linear force and the non-linear force causes the rms emittances and eigen-emittances to grow. Once the beam enters the analyzing magnet the horizontal rms emittance starts to increase gradually, but the vertical rms emittance is not changed. After charge selection, if the collimators are not adopted to cut particles, the rms emittances and eigen-emittances are almost constant until the first skew quadrupole. If the collimators are adopted, utilization of collimators stepwise decreases the rms emittances and eigen-emittances in the matching section. Inside of the skew quadrupole triplet, rms emittances are made equal to the separated eigen-emittances.

The behaviors of dimensionless brilliances and the transmission efficiencies along beam line with (horizontal half slit width $h=2 \mathrm{~cm}$ and vertical half slit width $v=2 \mathrm{~cm}$ ) and without collimators are shown in Fig. 1.

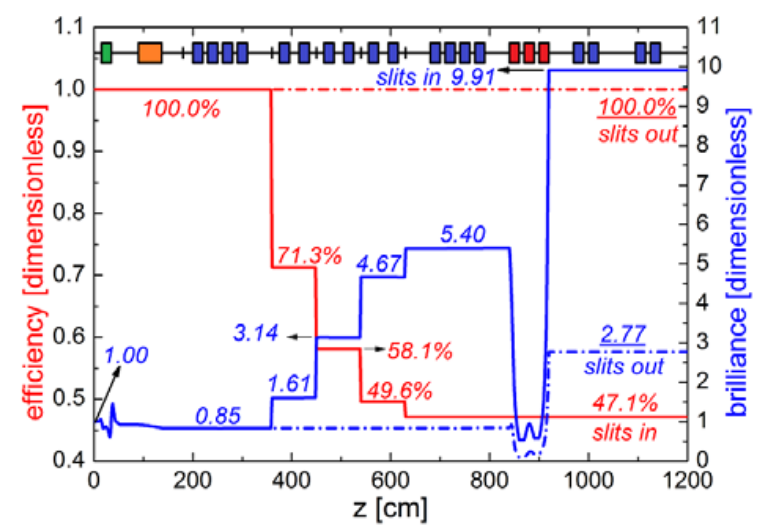

Figure 1: Dimensionless brilliance and transmission efficiency evolutions along the beam line with (solid lines) and without (dash lines) four successive slit collimators.

\section{References}

[1] C. Xiao, L. Groening and O.K. Kester, NIM-A, 2014, 738: p. 167-176.

[2] C. Xiao, L. Groening and O.K. Kester, Phys. Rev. ST Accel. Beams 16, 044201 (2013). 